University of Nebraska - Lincoln

DigitalCommons@University of Nebraska - Lincoln

$1-2014$

\title{
Experimental and numerical investigation of carbon fiber sandwich panels subjected to blast loading
}

\author{
Yi Hua \\ University of Nebraska-Lincoln, yhua3@unl.edu \\ Praveen Kumar Akula \\ University of Nebraska-Lincoln \\ Linxia Gu \\ University of Nebraska-Lincoln, gul@fit.edu
}

Follow this and additional works at: https://digitalcommons.unl.edu/mechengfacpub

Hua, Yi; Akula, Praveen Kumar; and Gu, Linxia, "Experimental and numerical investigation of carbon fiber sandwich panels subjected to blast loading" (2014). Mechanical \& Materials Engineering Faculty Publications. 96.

https://digitalcommons.unl.edu/mechengfacpub/96

This Article is brought to you for free and open access by the Mechanical \& Materials Engineering, Department of at DigitalCommons@University of Nebraska - Lincoln. It has been accepted for inclusion in Mechanical \& Materials Engineering Faculty Publications by an authorized administrator of DigitalCommons@University of Nebraska Lincoln. 


\title{
Experimental and numerical investigation of carbon fiber sandwich panels subjected to blast loading
}

\author{
Yi Hua, ${ }^{1}$ Praveen Kumar Akula, ${ }^{1}$ and Linxia Gu ${ }^{1,2}$ \\ 1. Department of Mechanical and Materials Engineering, University of Nebraska-Lincoln, Lincoln, NE 68588-0656, USA \\ 2. Nebraska Center for Materials and Nanoscience, Lincoln, NE 68588-0656, USA \\ Corresponding author - L. Gu, Department of Mechanical and Materials Engineering, University of Nebraska-Lincoln, \\ Lincoln, NE 68588-0656, USA; tel 402 472-7680, fax 402 472-1465, email lgu2@unl.edu
}

\begin{abstract}
The objective of this paper is to investigate the structural response of carbon fiber sandwich panels subjected to blast loading through an integrated experimental and numerical approach. A total of nine experiments, corresponding to three different blast intensity levels were conducted in the 28-inch square shock tube apparatus. Computational models were developed to capture the experimental details and further study the mechanism of blast wave-sandwich panel interactions. The peak reflected overpressure was monitored, which amplified to approximately 2.5 times of the incident overpressure due to fluid-structure interactions. The measured strain histories demonstrated opposite phases at the center of the front and back facesheets. Both strains showed damped oscillation with a reduced oscillation frequency as well as amplified facesheet deformations at the higher blast intensity. As the blast wave traversed across the panel, the observed flow separation and reattachment led to pressure increase at the back side of the panel. Further parametric studies suggested that the maximum deflection of the back facesheet increased dramatically with higher blast intensity and decreased with larger facesheet and core thickness. Our computational models, calibrated by experimental measurements, could be used as a virtual tool for assessing the mechanism of blast-panel interactions, and predicting the structural response of composite panels subjected to blast loading.
\end{abstract}

Keywords: Shock tube testing, A. Layered structures, B. Impact behavior, B. Interface/interphase, C. Finite element analysis

\section{Introduction}

Sandwich panels, which consist of two thin facesheets adhered to a thick core, are increasingly used in blast protections due to their high specific stiffness and strength, as well as superior energy absorbing capacity [1]. In recent years, attention has been drawn from the blast loading response of monolithic structures [2-7] to that of sandwich panels. Dharmasena et al. [8] conducted explosive testing in the air to study the dynamic response of sandwich panels made of super-austenitic stainless steel alloy. They observed that the sandwich panel had a lower back facesheet deflection than the monolithic plate, and the advantages of sandwich panels were diminished after complete core crushing. Fleck and Deshpande [9] theoretically studied the dynamic response of steel sandwich beams subjected to air and underwater blast loading, and developed performance charts of the sandwich beams with different core materials. Zhu et al. [10] studied the blast loaded aluminum sandwich panel with a cellular core. The effects of plastic deformation and clamped vs. simply supported boundary conditions on the back facesheet deflection were presented through finite element modeling. Karagiozova et al. [11] numerically analyzed the behavior of clamped steel sandwich panels with the surface pressure history mimicking the blast loading situation, and stated that the load transfer to the back facesheet of the panel with specific core material depended on the load intensity, core thickness and flexibility of sandwich panels. The blast resistance of E-glass fiber sandwich panels with stitched foam core [12] or stepwise graded core [13], were studied through the shock tube experiments. The recorded transient displacement and the damaged sandwich panels resulted from blast loadings were compared. The aforementioned studies mainly focus on the blast load response of sandwich panels with metal facesheets. The investigations of composite sandwich panels are limited [12, 13], even though they are frequently used in various engineering constructions [14-16]. Moreover, the repeatability of experimental results has seldom been ensured and few attempts have been made to investigate the structural response of sandwich panels using strain measurement techniques $[17,18]$.

In this work, the structural response of carbon fiber sandwich panels subjected to blast loading was investigated using an integrated experimental and numerical approach. A total of nine experiments, corresponding to three different blast intensity levels (low, medium and high), were conducted inside our shock tube apparatus. To further elucidate the mechanism 


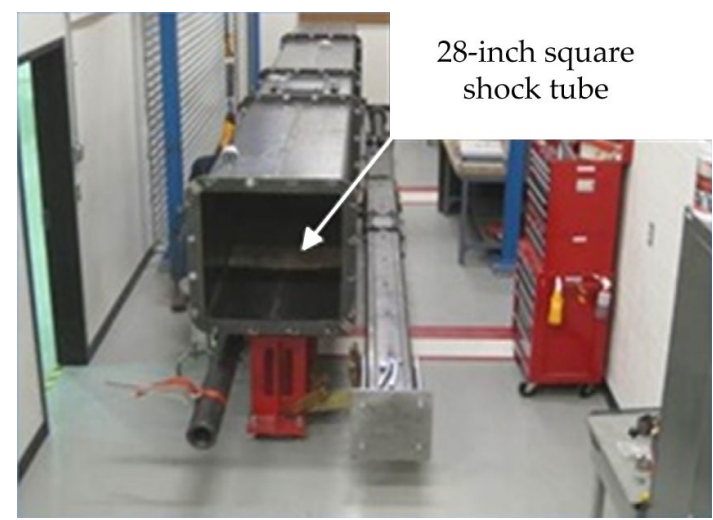

Figure 1. A $711 \mathrm{~mm}\left(28^{\prime \prime}\right)$ square shock tube apparatus.

of blast wave-sandwich panel interactions, a 3D finite element (FE) model was developed to reproduce the shock tube experiment, and then calibrated by the measured pressure profiles and strains on the facesheets. Finally, a parametric study was carried out to examine the impact of blast intensity and panel geometry on the maximum deflection of the sandwich panel back facesheet.

\section{Experimental procedure and results}

\subsection{Experimental procedure}

A $711 \mathrm{~mm}$ or $28^{\prime \prime}$ square shock tube apparatus with a length of $10 \mathrm{~m}$ (Figure 1) was used to create the controllable blast loading. Detailed description of the shock tube and its calibration can be found in [19]. Briefly, the square shock tube consisted of four main components including the driver, transition and straight sections, as well as the catch tank. The straight section was divided into a test region and an extension region. The driver section contained pressurized gas which was separated from the transition section by several membranes. As membranes ruptured due to increased gas pressure, the rapid release of gas produced a shock wave, which travel down the transition and extension sections and then interact with the specimen placed in the test section. Finally, the shock wave exited the shock tube and entered the catch tank which absorbed and slowly released most of the shock energy and reduced the noise intensity.
The $146 \mathrm{~mm}$ square sandwich panel, with four holes drilled close to the edges, was clamped by two L-shaped steel frames as specified in Figure 2. The frames were then fixed onto the bottom of the test section in shock tube. The sandwich panel (CST Inc., Tehachapi, CA) used in the shock tests consisted of two facesheets with a thickness of $0.762 \mathrm{~mm}$ each and a foam core with a thickness of $6.35 \mathrm{~mm}$. Rohacell 71 IG polyurethane (PMI) rigid foam was used as the core material, which was $100 \%$ closed cell and had constant shear strength through the thickness. The facesheets were fabricated from six-ply unidirectional carbon fiber prepreg tape $\left(150 \mathrm{~g} / \mathrm{m}^{2}\right.$ fiber areal weight and $35 \mathrm{wt} \%$ resin content) with a fiber orientation of $0-90^{\circ}$ in alternating layers and cured at 250F onto the PMI cores.

Vishay SR-4 general-purpose strain gauges with a grid resistance of $350 \pm 0.3 \% \Omega$ and a gauge factor of $2.09 \pm 0.5 \%$ were bonded at the center of front and back facesheets and connected to a Wheatstone quarter bridge to measure the transverse strain. Two piezoelectric pressure sensors (PCB 134A24) were used to record both the incident and reflected pressure histories. The sensor for measuring the incident pressure was mounted on the side wall of the shock tube with an offset of $0.2 \mathrm{~m}$ in front of the specimen, while the reflected pressure was measured by a sensor glued close to the right edge of the front facesheet, as labeled in Figure 2. Three different blast intensity levels, referred to as low, medium and high, were generated by rupturing a stack of 2, 6 and 10 plies of $0.025 \mathrm{~mm}$ thick Mylar membranes. For each level of blast intensity, three repeated experiments were conducted on the same panel.

\subsection{Experimental results and discussions}

\subsubsection{Characterization of the incident and reflected waves}

An important requirement of this study was the ability to produce repeatable and measureable blast loading conditions. The measured incident and reflected parameters were summarized in Table 1 . The peak overpressure was determined by using a 100-point average of the maximum pressure values after the arrival of the shock front. This was typically about $8 \mu \mathrm{s}$ after the shock arrival, which corresponded to the time for the shock to cross the sensor tip. The positive duration was referred to the time period with positive overpressure. The maximum impulse was calculated as the pressure-time integral over the entire positive duration. It was clear that both incident peak overpressure and maximum impulse increased with

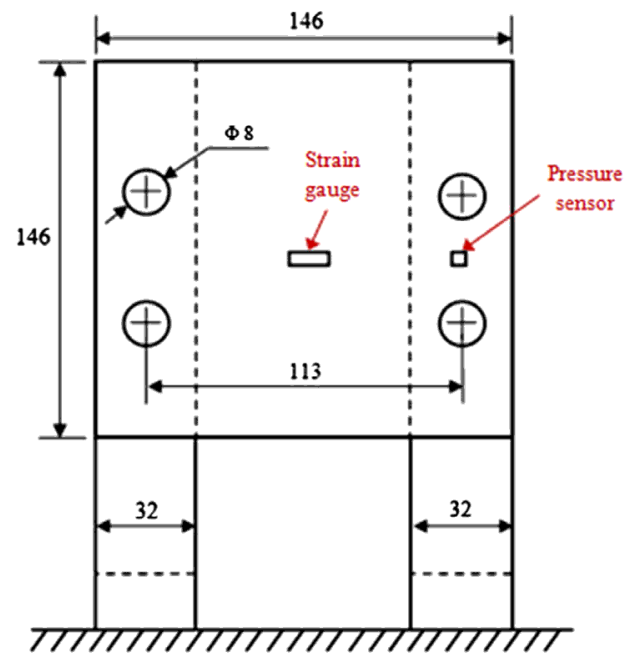

Front view

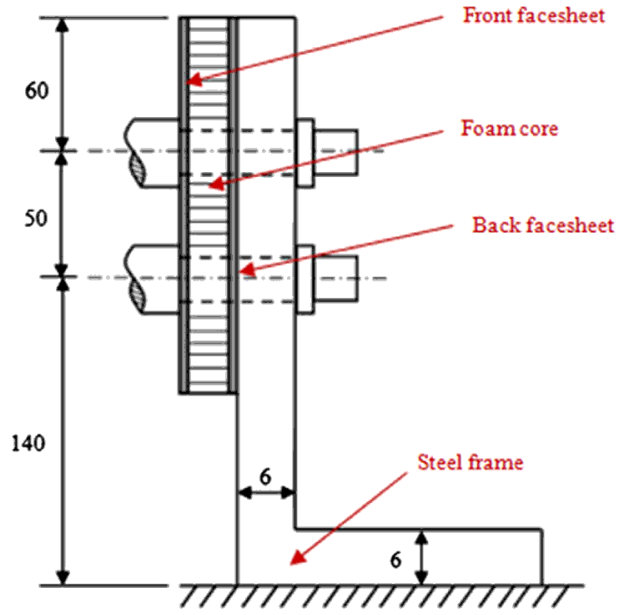

Side view

Figure 2. Sketch of the clamping sandwich panel. All dimensions are in $\mathrm{mm}$. 
Table 1. Summary of the measured incident and reflected parameters (average value and standard deviation).

\begin{tabular}{|c|c|c|c|}
\hline Blast intensity & Low & Medium & High \\
\hline \multicolumn{4}{|c|}{ Incident parameters } \\
\hline Peak overpressure (MPa) & $0.0658 \pm 0.0043$ & $0.1411 \pm 0.0048$ & $0.2022 \pm 0.0057$ \\
\hline $\begin{array}{l}\text { Maximum impulse } \\
\text { (MPa ms) }\end{array}$ & $0.108 \pm 0.002$ & $0.219 \pm 0.008$ & $0.305 \pm 0.007$ \\
\hline Positive duration (ms) & $4.1569 \pm 0.2410$ & $4.2578 \pm 0.0345$ & $4.3757 \pm 0.1078$ \\
\hline \multicolumn{4}{|c|}{ Reflected parameters } \\
\hline Peak overpressure (MPa) & $0.1657 \pm 0.0138$ & $0.4279 \pm 0.0396$ & $0.6513 \pm 0.0371$ \\
\hline $\begin{array}{l}\text { Maximum impulse } \\
\quad(\mathrm{MPa} \mathrm{ms})\end{array}$ & $0.129 \pm 0.008$ & $0.299 \pm 0.024$ & $0.464 \pm 0.011$ \\
\hline Positive duration (ms) & $3.9387 \pm 0.2326$ & $4.3008 \pm 0.2383$ & $4.2962 \pm 0.1357$ \\
\hline
\end{tabular}

the higher level of blast intensity. Considering the low blast intensity as a benchmark, the incident peak overpressure increased 2.1 times and 3.1 times, respectively, for the medium and high blast intensity, while the transmitted maximum impulse increased 2.0 times and 2.8 times, respectively. The positive duration of the incident pressure was not sensitive to the blast intensity and only had a maximum $5.26 \%$ increase as the blast intensity varied from low to high.

Compared with an incident overpressure, a much larger reflected peak overpressure was obtained. The ratio of reflected peak overpressure to incident peak overpressure was referred to as the reflection factor. It was observed as 2.5, 3.0, and 3.2 for low, medium, and high blast intensity, respectively. This pressure amplification behavior was due to the fluid-structure interaction (FSI). The reflection ratio can vary from 2 to 8 , depending on several factors like the incident blast intensity, fluid medium in which the blast wave travels, angle of incidence, mass and geometry of the object [20]. The impulse amplification was also observed. The reflected maximum impulse was $19.44 \%, 36.53 \%$ and $52.13 \%$ larger than that of the incident one under low, medium and high blast intensity, respectively. In contrast, the reflected positive duration remained almost unchanged compared with that of the incident one. These indicated that the reflected peak overpressure was more sensitive to the blast wave-sandwich panel interface than the transmitted maximum impulse and positive duration.

Considering the repeatability of experimental measures shown in Table 1, one representative incident and reflected pressure profiles for each blast intensity level were depicted in Figure 3. It is observed that both the sharp rise and exponential decay of the incident pressure histories followed a typical Friedlander 1D shock wave given by Baker [21]

$$
p(t)=p_{0}+P_{s}^{+}\left(1-\frac{t}{T^{+}}\right) e^{-b t / T^{+}}
$$

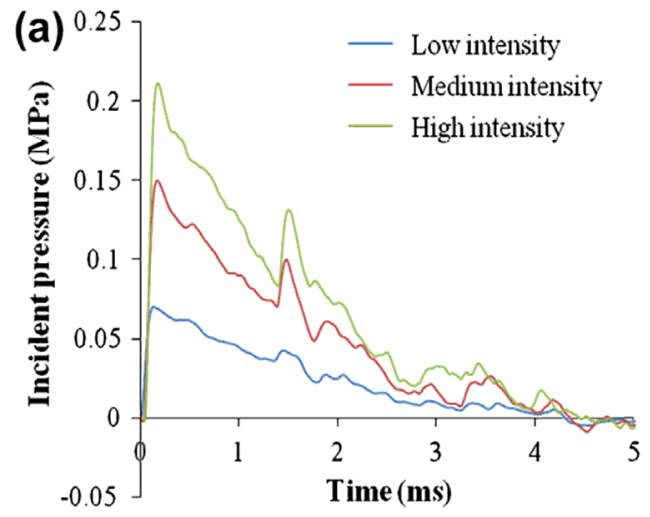

where $p$ denoted pressure; $t$, time; $p_{0}$, ambient pressure; $P_{s}^{+}$ peak overpressure; $T^{+}$, positive phase duration and $b$, decay constant. A set of small secondary peaks could be attributed to the reflections from the sandwich panel; however, these reflections did not significantly affect the pressure profiles. The measured reflected overpressure profiles, qualitatively similar to the incident ones, were shown in Figure 3(b). The notable difference was the faster pressure decay.

\subsubsection{Strain histories measured at the center of the front and back facesheets}

Strain histories measured at the center of the front and back facesheets under three different blast intensity levels were shown in Figure 4(a-c), respectively. It was observed that the strain history profiles oscillated in a damped sinusoidal manner and the strains at the front and back facesheets were opposite to each other. As the blast wave hit the panel, the front facesheet experienced compression while the back facesheet underwent tension. The elasticity drove the backwards motion of the sandwich panel, which then oscillated in an elliptic manner. The peak oscillation frequencies were calculated using Fourier transforms as approximately $1000 \mathrm{~Hz}, 500 \mathrm{~Hz}$ and $400 \mathrm{~Hz}$ for low, medium and high blast intensity, respectively. It is also clear that higher blast intensity led to a reduced oscillation frequency, and an increased facesheet deformation. For example, the maximum transverse strain at the front and back facesheets was $0.704 \%$ and $0.594 \%$, respectively for the high blast intensity, while it was only $0.125 \%$ and $0.096 \%$ for the low one. In addition, the peak strain at the back facesheet was a little smaller than that at the front one. This could be due to the energy dissipation in the core material.

\section{Finite element modeling}

To further understand the detailed mechanism of blast wavesandwich panel interactions, FE model capturing the blast tests (Figure 5) was developed using commercial software ABAQUS (Dassault Systems Simulia Corp., RI, USA). The composite facesheets, as described in Section 2.1, were characterized by orthotropic material with the orthotropic stiffness constants $D_{11_{11}}=D_{2222}=75,718 \mathrm{MPa}, D_{3333}=12,450 \mathrm{MPa}$, $D_{1122}=8511.9 \mathrm{MPa}, D_{1133}=D_{2233}=7464.7 \mathrm{MPa}, D_{1212}=$ 2903.7 MPa, $D_{1313}=D_{2323}=2959.9 \mathrm{MPa}$. The foam core was assumed as isotropic material with Young's modulus $E=$ $92 \mathrm{MPa}$ and Poisson's ratio $v=0.16$. The mass density of the facesheets and foam core was specified as $1.52 \times 10^{3} \mathrm{~kg} / \mathrm{m}^{3}$ and $75 \mathrm{~kg} / \mathrm{m}^{3}$, respectively. Air was modeled as an ideal gas equation of state (EOS) since the Mach number of the shock front from our experiments was less than 2, and the ratio of specific heats did not change drastically at this Mach number [22].

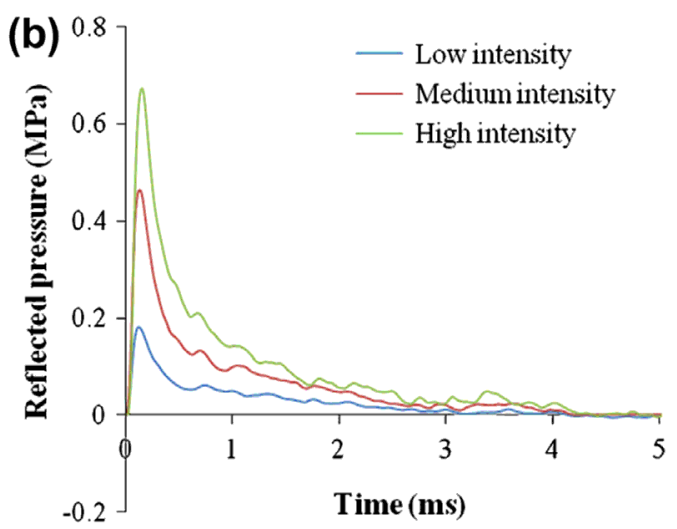

Figure 3. Measured pressure profiles: (a) incident pressure and (b) reflected pressure. 


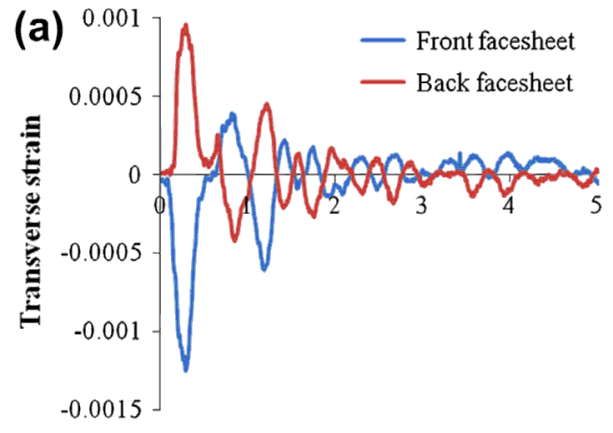

Time (ms)

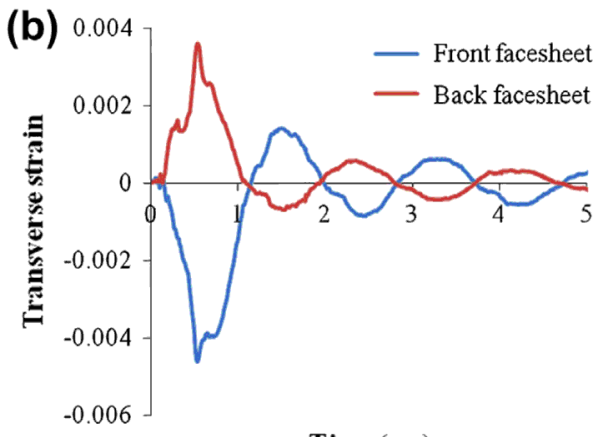

Time (ms)

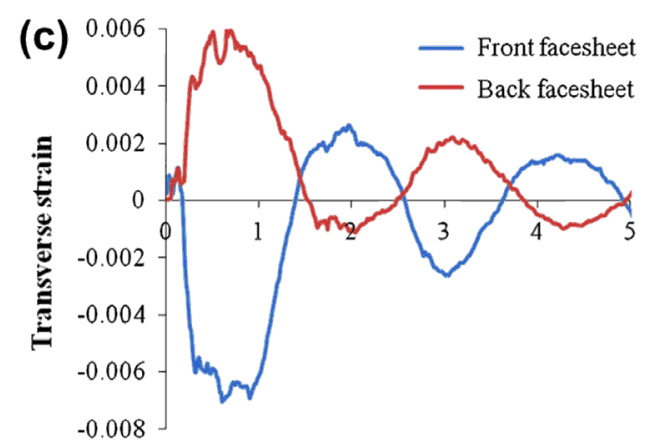

Time (ms)

Figure 4. Strain histories measured at the center of the front and back facesheets under three different blast intensities: (a) low, (b) medium and (c) high.

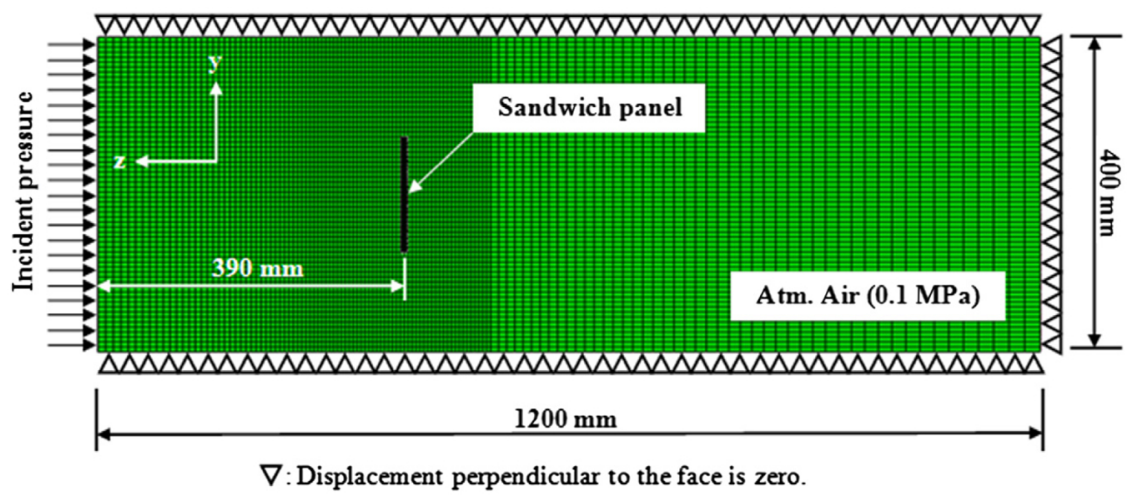

Figure 5. FE model of sandwich panel subjected to blast loading (cut view in transverse plane).

The blast wave propagation and its interaction with the sandwich panel is essentially a FSI problem. The main challenge is to capture the 2 ms shock event by ensuring an efficient coupling between fluid domain and solid domain. In this work, the air inside the shock tube was modeled with Eulerian elements, which could mimic the highly dynamic blast events. The sandwich panel was modeled with Lagrangian elements. The coupling was solved through the penalty contact algorithm with frictionless tangential sliding and hard contact normal behavior. Eulerian domain consisted of 511, 686 brick elements with approximate mesh refinement near the region of sandwich panel to capture FSI effects. To save the computation time, the size of the Eulerian domain was chosen as $400 \mathrm{~mm} \times 400 \mathrm{~mm} \times 1200 \mathrm{~mm}$ such that the reflections from domain boundaries were negligible during total $5 \mathrm{~ms}$ simulation time. The front and back facesheets of the sandwich panel were meshed with 4-node shell elements with finite membrane strains (S4R). Five integration points with Simpson's integration rule were used in each shell element. The foam core

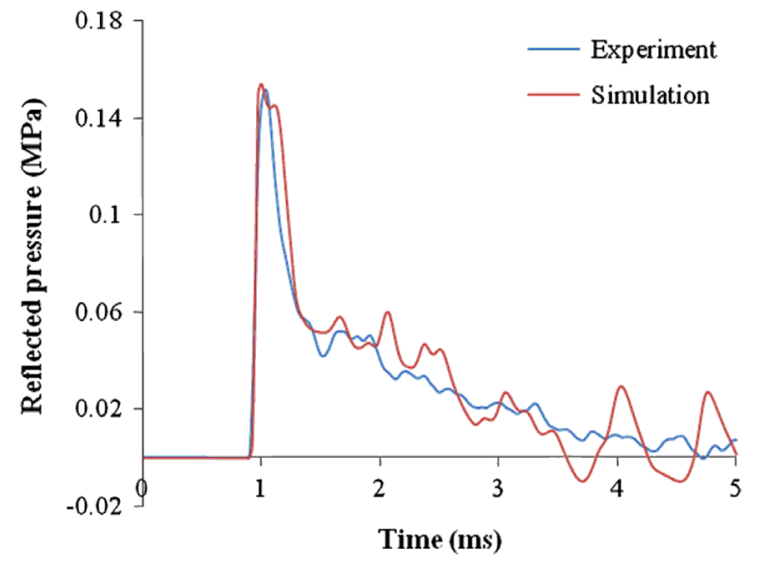

Figure 6. Comparison of reflected pressure histories from experiment and numerical simulation. 


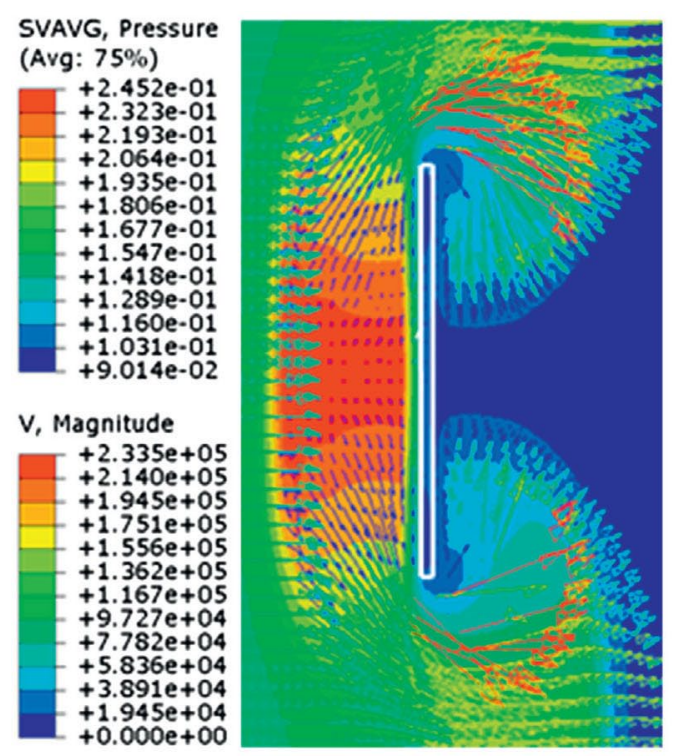

(a) $\mathrm{t}=1.15 \mathrm{~ms}$

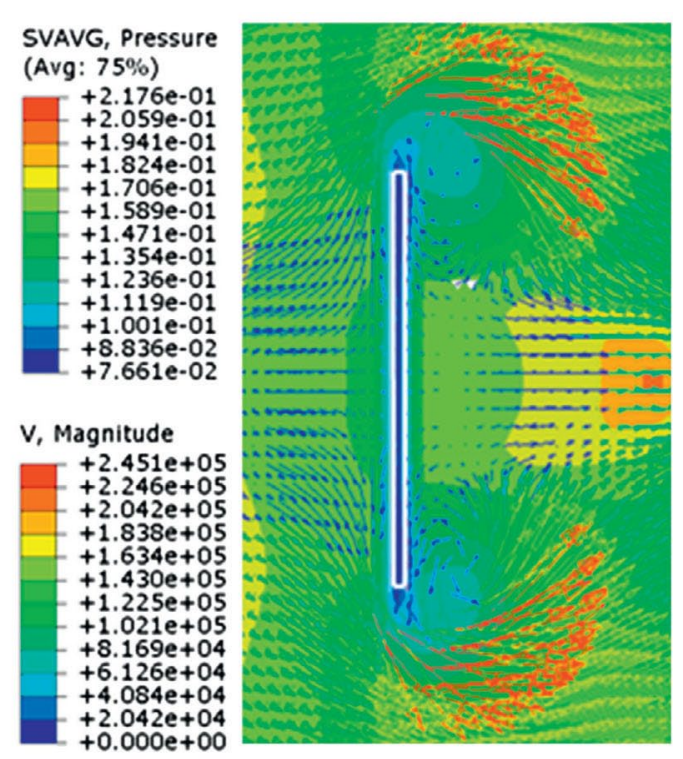

(b) $\mathrm{t}=1.35 \mathrm{~ms}$

Figure 7. Velocity profile overlaid on the pressure distribution around the sandwich panel to highlight: (a) the initial pressure relief and (b) the vortex formation and flow reattachment behind the panel.

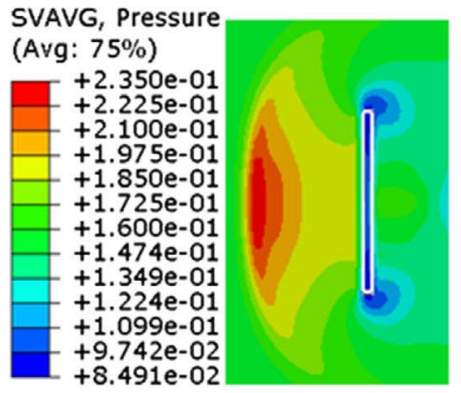

(a)

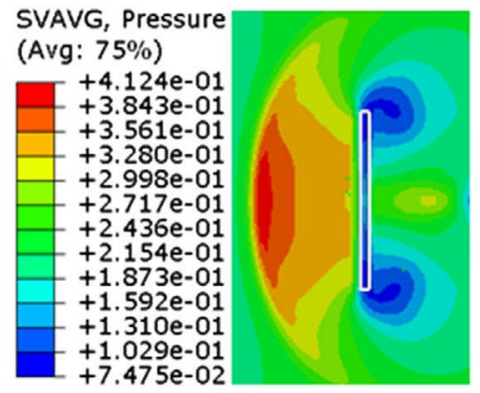

(b)

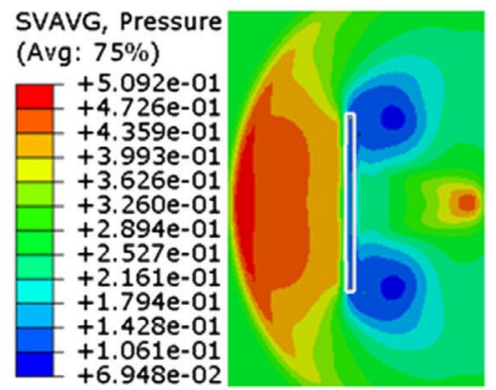

(c)

Figure 8. Flow separation for the blast intensity at (a) low (b) Medium and (c) High.

was fully meshed with reduced 8-node hexahedral elements (C3D8R). A mesh convergence test has been conducted and the minimum mesh size of both facesheets and foam core was chosen as $2 \mathrm{~mm}$.

The measured incident pressure history in Section 2.2.1 was used as the pressure boundary condition at the inlet of the Eulerian domain. The velocity perpendicular to each face of Eulerian domain was kept zero to avoid escaping/leaking of air through these faces. This would create a pure 1D shock front traveling in the $\mathrm{z}$-direction without lateral flow. To simulate the fixation of sandwich panel used in our experiment, the two margins (32 mm wide) of back facesheet were constrained in all six degrees of freedom to avoid rigid body translation. The tied constraint was used between two facesheets and foam core.

Table 2. Comparison of peak strain magnitude measured at the center of front and back facesheets from experiment and numerical simulation.

\begin{tabular}{lccc}
\hline & Experiment & Simulation & Deviation (\%) \\
\hline Front facesheet & -0.00125 & -0.0011 & 12.0 \\
Back facesheet & 0.00096 & 0.001 & 4.2 \\
\hline
\end{tabular}

\section{Simulation results and discussion}

\subsection{Simulated experiments}

FE results were compared with the measured parameters in the shock tube. For brevity we only focus on the case of low blast intensity in the following discussion. Figure 6 shows the reflected overpressure histories obtained from both the FE model and experimental measurement. For the comparison, the arrival time of the experimental measured pressure profile was shifted to match that of numerical simulation. It was clear that the major features of the measured overpressure profile, including the shock front rise time, exponential decay, and small peaks and valleys were captured by the simulation. The deviation of the peak overpressure was only $1.74 \%$, which could be attributed to the ideal gas EOS assumption, friction along the inner wall of the shock tube, and sensitivity of pressure sensors. Large amplitude vibration of the reflected pressure was observed from the simulation at the end of the decay period (from $3.5 \mathrm{~ms}$ to $5 \mathrm{~ms}$ ). This was due to the blast wave which reflected by the lateral surface of the Eulerian domain at that time. In addition to the overpressure, the peak strain at the center of front and back facesheets were also compared between the experimental measurements and numerical simulation, as listed in Table 2. The deviation of strains was $12.0 \%$ 

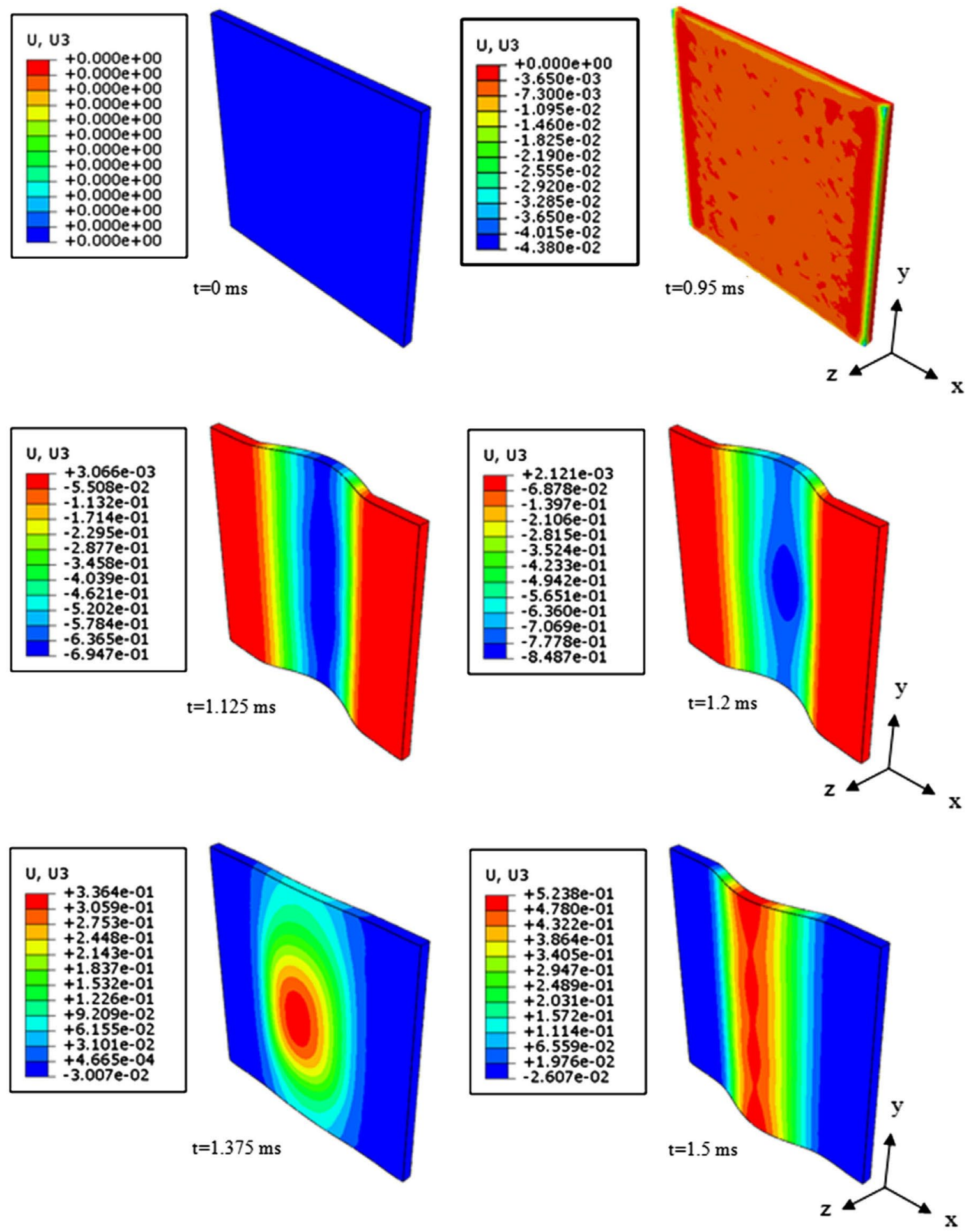

Figure 9. Snapshots of the deflections of the sandwich panel subjected to blast loading (deformation scale factor of 30).

and $4.2 \%$ for the front and back facesheets, respectively. This difference could be attributed to the misalignment between the strain gauge and the center of the facesheet, as well as the effect of hot blast temperature on the gauge sensitivity. We have demonstrated that the simulation strains as well as the overpressure profiles agreed with the corresponding experimental results. The calibrated model can then be used to gain insights into the detailed mechanism of blast wave-sandwich panel interactions.

\subsection{Blast wave-sandwich panel interactions}

As the blast wave hit the front facesheet of the sandwich panel, normal reflection occurred and the panel was instantly subjected to the reflected overpressure. The pressure distribution as well as the flow vector field in the vicinity of the sandwich panel was depicted in Figure 7. It is clear that the reflected pressure was substantially higher than the pressure surrounding the sandwich panel. Consequently, there was a flow of air 

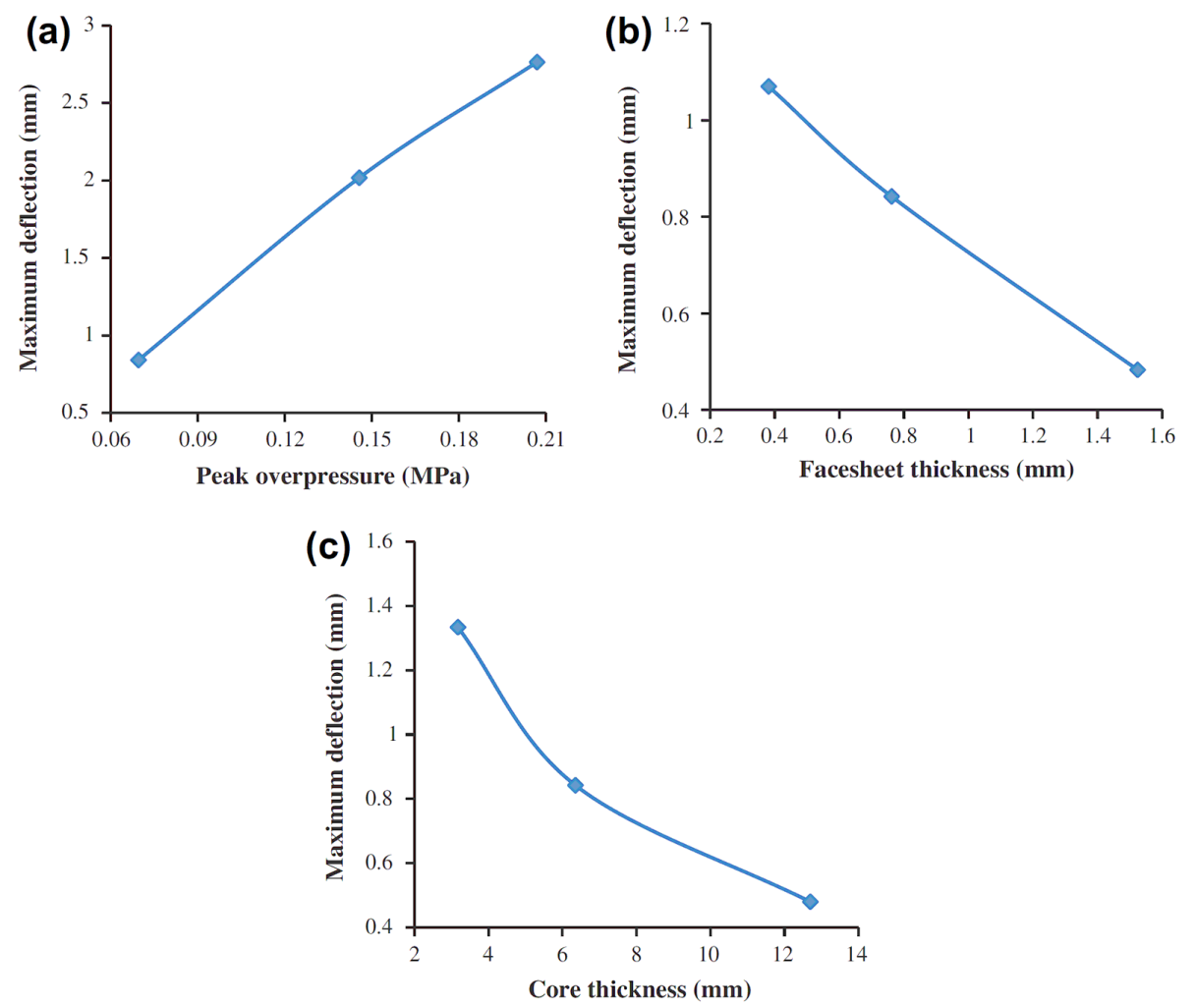

Figure 10. The maximum deflection of the back facesheet depends on (a) blast intensity, (b) facesheet thickness and (c) core thickness.

from the region of high pressure to the one at low pressure, as visualized by the flow vectors. It was observed that the velocity vectors at the front facesheet were parallel to the reflecting surface and pointing towards both the top and bottom edges, respectively. This parallel flow became detached from the surface at the leading edge, and partially diverged incident wave away from the panel edges, causing an instantaneous pressure relief and the vortices near the edges. During the formation of vortex, a significant pressure decrease was observed approximately at the center of vortex. This flow divergence, also referred to as flow separation, was magnified at higher blast intensity, with lower pressures within the vortex, as demonstrated in Figure 8. This qualitative observation agrees with the results from the theoretical studies on blast-structure interactions [23, 24]. Moreover, the separated blast waves reattached together behind the panel, resulting in an increase in pressure. It is also worth noting that the flow separation and vortex formation was closely related to the onset of the structure damage as the vortex flows induced by the much higher intensity blasts were strong enough to push the structures towards the low pressure zone within the vortex [24].

\subsection{Structural response of the sandwich panel}

The structural response of sandwich panel subjected to blast loading was shown in Figure 9. Due to the low-level blast intensity used in our simulation, there was no plastic deformation and failure occurred to the sandwich panel. The motion of the panel was considered forth (negative z-direction) or back (positive $z$-direction) with respect to its original flat shape at $t=0 \mathrm{~ms}$. At $t=0.95 \mathrm{~ms}$, the wave front propagated through the ambient air and hit the front facesheet of the sandwich panel. The stress wave did not propagate into the back facesheet yet, so there was only negative deflection observed. The kinetic energy from the reflected overpressure was then transmitted to the sandwich panel and its central region moved forth as de- picted at $t=1.125 \mathrm{~ms}$, and then reached to maximum negative deflection of $0.85 \mathrm{~mm}$ at $t=1.2 \mathrm{~ms}$. As time moving on, the central region deflection moved back due to elastic relaxations as demonstrated at $t=1.375 \mathrm{~ms}$. Continuing backward movement of the panel center led to the maximum positive deflection of $0.52 \mathrm{~mm}$ at time $t=1.5 \mathrm{~ms}$. However, this peak positive deflection was $38.1 \%$ smaller than the peak negative one at $t=1.2 \mathrm{~ms}$. As the time progressed, elastic vibrations took place until the deflection of the sandwich panel reverted to zero. During the whole process, the internal energy in the foam core was found to be 8.8 and 4.5 times of that in the front and back facesheets, indicating the energy absorption ability or blast resistance ability of the foam core.

The maximum back facesheet deflection is usually considered as the measure of the blast resistance. The role of the blast intensity, the facesheet and core thicknesses were studied to provide more insights into the design of sandwich structure. In our base model, the peak overpressure of the blast wave was set as $0.07 \mathrm{MPa}$, corresponding to the low blast intensity in our experiments, and the facesheet and core thicknesses were assumed as $0.762 \mathrm{~mm}$ and $6.35 \mathrm{~mm}$, respectively. Figure 10(a) shows the effect of blast intensity on the maximum deflection of the back facesheet. Three peak overpressures, i.e., $0.07 \mathrm{MPa}, 0.14 \mathrm{MPa}$ and $0.20 \mathrm{MPa}$, corresponding to the experimental setup (Table 1) have been used to calculate the maximum deflection of back facesheet. It was obvious that the maximum deflection of the back facesheet increased with higher peak overpressure. The maximum deflection increased 2.28 times when the peak overpressure increased from $0.07 \mathrm{MPa}$ to $0.20 \mathrm{MPa}$. However, the growth rate of the maximum deflection decreased with higher peak overpressure. It was observed that the maximum deflection increased 1.40 times by varying the peak overpressure from $0.07 \mathrm{MPa}$ to $0.14 \mathrm{MPa}$, compared to 0.37 times from $0.14 \mathrm{MPa}$ to $0.20 \mathrm{MPa}$. This indicated that the benefits of sandwich construction were particular evident at low blast intensity. 
The influence of facesheet thickness on the maximum deflection of the back facesheet was shown in Figure 10(b). Three different facesheet thicknesses were considered: $0.381 \mathrm{~mm}$, $0.762 \mathrm{~mm}$ and $1.524 \mathrm{~mm}$. As expected, sandwich panel with larger facesheet thickness proved to be more efficient in preventing blast. Compared with the facesheet of thickness of $0.381 \mathrm{~mm}$, the maximum deflection of the back facesheet decreased $21.3 \%$ and $54.9 \%$ when the facesheet thickness was $0.762 \mathrm{~mm}$ and $1.524 \mathrm{~mm}$, respectively. Figure 10(c) shows the influence of core thickness ( $3.175 \mathrm{~mm}, 6.35 \mathrm{~mm}$ and $12.7 \mathrm{~mm}$ ) on the maximum deflection of the back facesheet. It is clear that the maximum deflection of the back facesheet was significantly decreased with a thicker core layer. Compared with the core thickness of $3.175 \mathrm{~mm}$, the maximum deflection of the back facesheet decreased $36.9 \%$ and $64.0 \%$ when the core thickness increased to $6.35 \mathrm{~mm}$ and $12.7 \mathrm{~mm}$, respectively. Both a thicker facesheet and core layer could reduce the maximum deflection of the back facesheet, which could be explained by the increased bending stiffness of the structure.

\section{Conclusions}

In this work, the structural response of sandwich panels subjected to blast loading was investigated using an integrated experimental and numerical approach. The novelty of this work lies in the development of a 3D shock tube FE model which calibrated by experiments to elucidate the mechanism of blast wave-sandwich panel interactions, as well as the quantification of the structural response of sandwich panels using strain measurement technique.

Experimental results showed that the incident peak overpressure and maximum impulse increased with higher blast intensity, while the positive duration remained almost unchanged. A much larger reflected peak overpressure and maximum impulse, as well as faster pressure decay were observed in comparison to the incident overpressure profile. The deformation profiles in a damped sinusoidal manner were totally opposite for the front and back facesheets. The sandwich panel oscillated in an elliptic manner and the peak oscillation frequencies reduced with higher blast intensity.

The mechanism of blast wave-sandwich panel interactions was further investigated using FE models to extract more information from these blast tests. The pressure profiles and strain measures from the simulations agreed with the repeated experimental measurements, which calibrated the developed FE models. In addition, the detailed flow separation, vortex formation, and flow reattachment were observed when the blast wave traversed across the panel. Snapshots of panel deflection were used to illustrate the damping behavior of the panel in related to the shock front locations.

The influence of blast intensity and panel geometry on the maximum deflection of its back facesheet was further studied through a parametric analysis. It was observed that higher peak overpressure induced dramatically larger deflection on the back facesheet. The growth rate of the deflection decreased with the increased peak overpressure. This indicated that the benefits of sandwich panels were particular evident at low intensity blast. Sandwich panels with larger facesheet and core thicknesses proved to be more efficient in blast resistance. However, this would increase the panel dimension and weight. Compromise between these parameters need to be considered for an optimal design. The calibrated model in this work could provide a fundamental understanding of the mechanism of blast wave-sandwich panel interactions, and provide guidance for optimizing the performance of the composite materials under the extreme loading conditions.
Acknowledgments - This study is partially supported by the US Army Research Office (ARO), Contract No.W911NF-08-1-0483. The authors would like thank Mr. Aaron Alai for his assistance in blast experiments. The authors also thank Mr. Mitchell Fullerton for final grammar check.

\section{References}

[1] Chi Y, Langdon GS, Nurick GN. The influence of core height and face plate thickness on the response of honeycomb sandwich panels subjected to blast loading. Mater Des 2010;31(4):1887-99.

[2] Jacinto AC, Ambrosini RD, Danesi RF. Experimental and computational analysis of plates under air blast loading. Int J Impact Eng 2001;25(10):927-47.

[3] Stoffel M, Schmidt R, Weichert D. Shock wave-loaded plates. Int J Solids Struct 2001;38(42-43):7659-80.

[4] Langdon GS, Schleyer GK. Inelastic deformation and failure of profiled stainless steel blast wall panels. Part I: experimental investigations. Int J Impact Eng 2005;31(4):341-69.

[5] Langdon GS, Schleyer GK. Inelastic deformation and failure of profiled stainless steel blast wall panels. Part II: analytical modelling considerations. Int J Impact Eng 2005;31(4):371-99.

[6] Rajendran R, Lee JM. Blast loaded plates. Mar Struct 2009;22(2):99-127.

[7] Kumar P, LeBlanc J, Stargel DS, Shukla A. Effect of plate curvature on blast response of aluminum panels. Int J Impact Eng 2012;46:74-85.

[8] Dharmasena KP, Wadley HNG, Xue ZY, Hutchinson JW. Mechanical response of metallic honeycomb sandwich panel structures to high-intensity dynamic loading. Int J Impact Eng 2008;35(9):1063-74.

[9] Fleck NA, Deshpande VS. The resistance of clamped sandwich beams to shock loading. J Appl Mech-T ASME 2004;71(3):386-401.

[10] Zhu F, Zhao LM, Lu GX, Gad E. A numerical simulation of the blast impact of square metallic sandwich panels. Int J Impact Eng 2009;36(5):687-99.

[11] Karagiozova D, Nurick GN, Langdon GS. Behaviour of sandwich panels subject to intense air blasts - Part 2: numerical simulation. Compos Struct 2009;91(4):442-50.

[12] Tekalur SA, Bogdanovich AE, Shukla A. Shock loading response of sandwich panels with 3-D woven E-glass composite skins and stitched foam core. Compos Sci Technol 2009;69(6):736-53.

[13] Wang EH, Gardner N, Shukla A. The blast resistance of sandwich composites with stepwise graded cores. Int J Solids Struct 2009;46(18-19):3492-502.

[14] LeBlanc J, Shukla A, Rousseau C, Bogdanovich A. Shock loading of three-dimensional woven composite materials. Compos Struct 2007;79(3):344-55.

[15] Fatt MSH, Palla L. Analytical modeling of composite sandwich panels under blast loads. J Sandw Struct Mater 2009;11(4):357-80.

[16] Andrews EW, Moussa NA. Failure mode maps for composite sandwich panels subjected to air blast loading. Int J Impact Eng 2009;36(3):418-25.

[17] Wang ZH, Jing L, Ning JG, Zhao LM. The structural response of clamped sandwich beams subjected to impact loading. Compos Struct 2011;93(4):1300-8.

[18] Shen JH, Lu GX, Wang ZH, Zhao LM. Experiments on curved sandwich panels under blast loading. Int J Impact Eng 2010;37(9):960-70.

[19] Kleinschmit NN, A shock tube technique for blast wave simulation and studies of flow structure interactions in shock tube blast experiments, Master thesis, University of Nebraska-Lincoln, USA, 2011.

[20] Anderson JD. Fundamentals of aerodynamics. New York: McGraw-Hill; 2001.

[21] Baker WE. Explosions in air. Austin: University of Texas Press; 1973.

[22] Ganpule S, Gu L, Alai A, Chandra N. Role of helmet in the mechanics of shock wave propagation under blast loading conditions. Comput Methods Biomech Biomedical Eng. 2012;15(11):1233-44.

[23] Kinney GF, Graham KJ. Explosive shocks in air. New York: Macmillan; 1962.

[24] Needham CE. Blast waves. New York: Springer; 2010. 